\title{
A diagnostic approach for identifying defects in the rhinobase
}

\author{
K. Dzhambazov, Topalova A. \\ Department of Otorhinolaryngology at University Hospital St. George, Plovdiv \\ Medical University of Plovdiv
}

\begin{abstract}
:
Introduction: Rhinoliquorrhea is a condition characterized by a cerebrospinal fluid leak through the nasal cavity due to a bone or dural defect in the rhinobase. It can be associated with serious lifethreatening conditions such as ascending bacterial infections from the non-sterile nasal cavity to the subarachnoid space - meningitis, meningoencephalitis, brain abscess, and others. The diagnostic approach for identifying rhinoliquorrhea is multidisciplinary and depends on the knowledge of different specialists - otolaryngologists, neurosurgeons, general practitioners, radiologists.

Materials and methods: For the 2015 - 2019 period 23 patients were diagnosed with rhinoliquorrhea at the Department of Otorhinolaryngology, University Hospital St. George, Plovdiv. They were verified by glucose and Beta-2 transferrin tests. We performed computed tomography, magnetic resonance imaging and cisternography. In cases of a negative CT finding for defects in the rhinobase but clinically positive, we used the method of applying fluorescein intrathecally with intraoperative positivity at the site of the defect.

Results: We confirmed pre-operatively that all patients have rhinoliquorrhea, though the localization of the defect in 3 of them differed intraoperatively. In one of the clinical cases, the topical diagnosis of rhinoliquorrhea was not possible despite the positive result of Beta-2 transferrin. Intraoperatively, we detected many small defects in the rhinobase area and, in some cases, meningocele in the area of the olfactory rhyme. For most patients the hospital stay lasted for 7 days, while one patient had an intracranial complication - a brain abscess. In all patients we used an endonasal endoscopic approach to surgically treat the defects in the rhinobase.

Conclusion: The diagnostic approach for identifying defects in the rhinobase requires knowledge of the symptom "rhinoliquorrhea", confirmation of its presence by biochemical tests, and detection the osteodural defect by imaging. The diagnostic difficulties arise from the diversity in the etiology, the topic and the medical history of the patient. A long-undetected rhinoliquorrhea may have fatal consequences for the patient, thus making it a socially significant problem.
\end{abstract}

Keywords: rhinoliquorrhea, CSF leak, rhinobase, skull base, beta-2 transferrin, osteodural defect

\section{Introduction:}

Rhinoliquorrhea is a pathological process characterized by a cerebrospinal fluid leak through the nasal cavity and the paranasal sinuses. The pathological communication between the sterile subarachnoid space and the flora of the sinonasal cavity is a prerequisite for the development of infections in the central nervous system, pneumocephalia, altered state of consciousness, even death in cases of major defects. The timely topical diagnosis of rhinoliquorrhea plays an important role in the therapeutic behavior and judgment of the volume and type of surgical approach in the area of the anterior skull base.

Willis described the first case of rhinoliquorrhea as early as the 17th century, followed by Miller who demonstrated a defect of the rhinobase during an autopsy in 1826. In 1923, Dandy published the first successful reconstruction of the skull base with rhinoliquorrhea, using a bicoronal craniotomy and placing a graft from fascia lata. The imperfections of Dandy's intracranial approach, which are associated with a high rate of intracranial complications - hemorrhage, cerebral edema, anosmia, memory loss, osteomyelitis of the bone graft, with a high rate of mortality and recurrence, have created conditions for the development of other surgical approaches with a higher success rate and a lower rate of mortality and morbidity. In 1948, Dohlman published the first case in the literature of an extra- 
cranial surgical approach for rhinoliquorrhea, followed by Wigand's endoscopic approach in 1981 . In the last three decades, the endoscopic endonasal approach has been established as the gold standard for treating rhinoliquorrhea. However, its usage is closely related to the accurate topical diagnosis of rhinoliquorrhea, which can be a serious diagnostic problem.

\section{Materials and methods}

For the 2015-2019 period at the Department of Otorhinolaryngology, University Hospital St. George, Plovdiv 23 patients were diagnosed with rhinoliquorrhea. They were verified by glucose and Beta-2 transferrin tests. We performed computed tomography, magnetic resonance imaging/ cisternography. In cases of a negative CT finding for defects in the rhinobase but clinically positive, we used the method of applying fluorescein intrathecally with intraoperative positivity at the site of the defect. Considering the risk of its use, we complied with a protocol for its implementation which has been agreed by both the Ethics Committee of the Medical University of Plovdiv and the Department of Anesthesiology and Intensive Care at the University Hospital St. George, Plovdiv. The high diagnostic value of fluorescein was assessed by the inconsistency of preoperative and intraoperative diagnostics.

\section{Results}

In all patients, we performed a high-resolution computed tomography (HRST) with a $0.6 / 1 \mathrm{~mm}$ slice and additional bone reconstructions. According to esteemed authors (Oakley, Cem Meco; Zapalac et al.), a high-resolution computed tomography is the first choice in the topical diagnosis of rhinoliquorrhea. In 20 of the clinical cases, we had confirmatory intraoperative localization relative to the HRCT result. In eleven of the patients we used additional diagnostic methods for recurrent duraplasty. Magnetic Resonance Imaging/Cisternography has been used in cases of spontaneous rhinoliquorrhea, suspected for empty sella, history of previous duraplasties, intracranial and pituitary tumors. In cases of suspected and uncertain defects with previous surgical interventions, we used $10 \%$ fluorescein intrathecally. In the Republic of Bulgaria, fluorescein is not authorized for intrathecal application (it is not authorized by the Food and Drug Administration/FDA). Therefore, we inform the patients about both the side effects and risks and the positives of using the method, and then they sign an informed consent, which is legally supported under the Unauthorized Medicines Act in the Republic of Bulgaria. The method is approved by the Ethical Committee of the Medical University of Plovdiv. The positivity of the defect when using fluorescein is statistically successful in $99 \%$ of the cases.

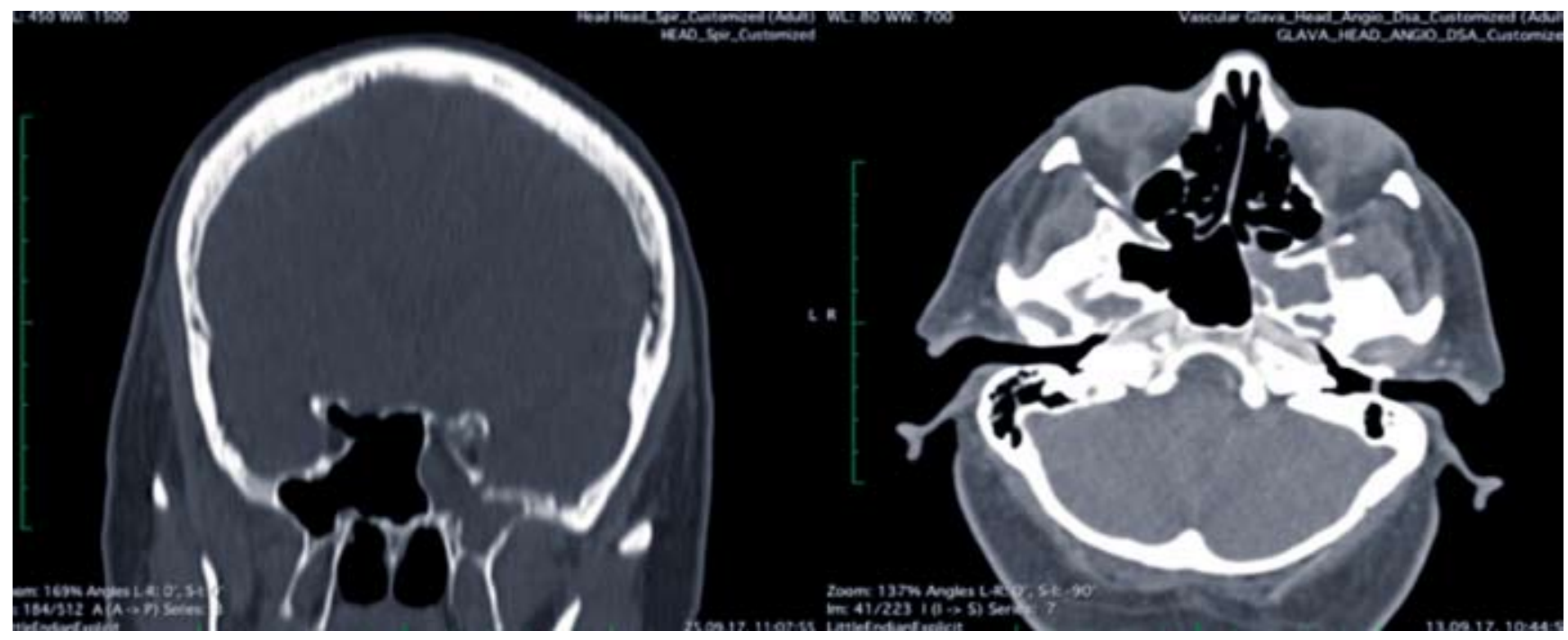

Figure 1. A female patient with spontaneous rhinoliquorrhea for 10 years. Clinical history of four meningitides. Treated for allergic rhinitis. CT data for a defect in the area of the lateral recess of the sphenoid sinus. Suspected area for Sternberg's Canal. Negative findings during the first endoscopic endonasal surgery. 


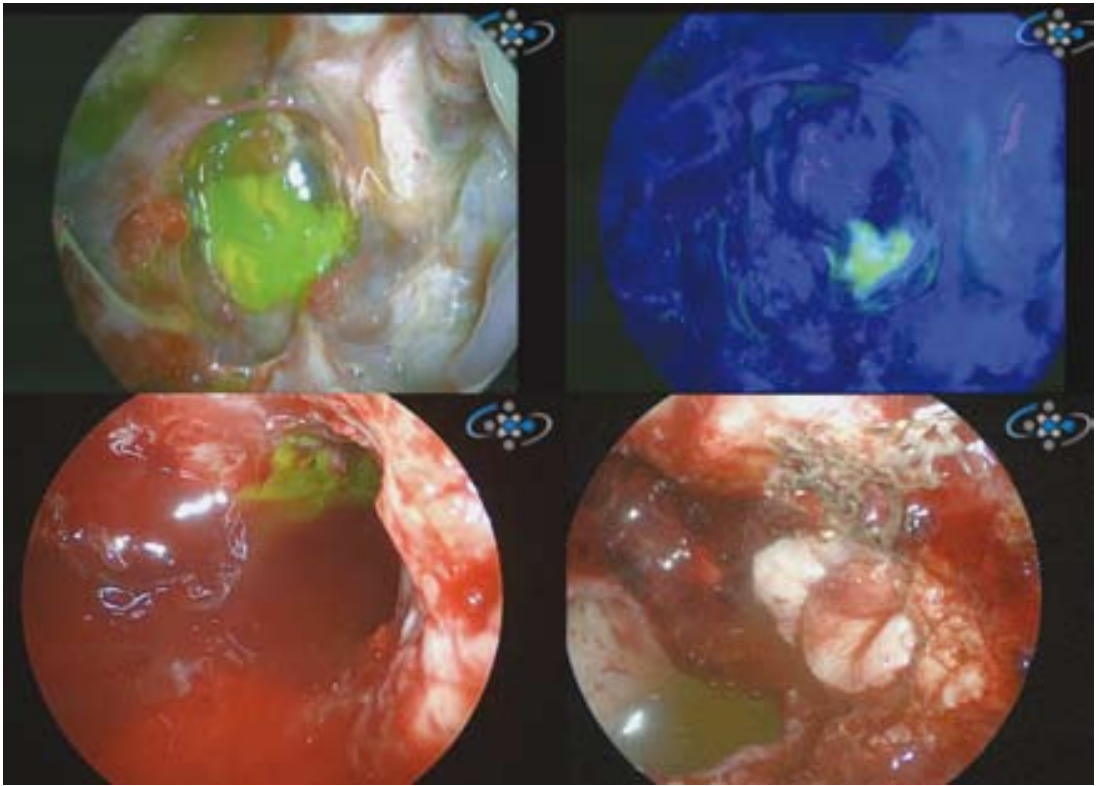

Figure 2. After the intrathecal application of fluorescein during the revision endoscopic endonasal duraplasty, several small defects are detected in the area of the sphenoethmoid recess, and in this case the sphenoid sinus plays the role of a "reservoir".

Performing bone reconstruction of a $0.6 \mathrm{~mm}$ slice has a high diagnostic value when there is a directional examination and good orientation by the radiologist.

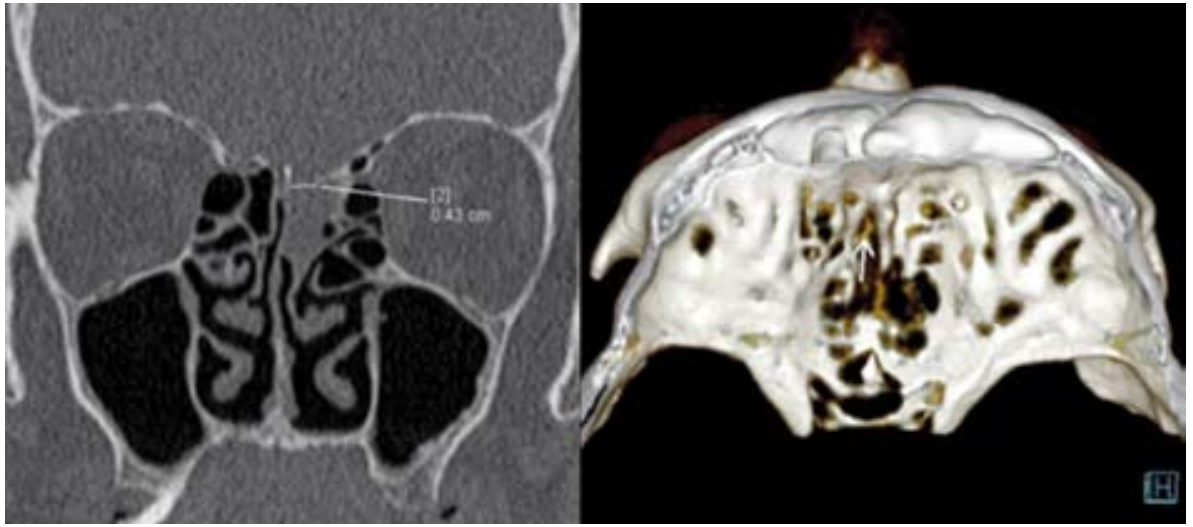

Figure 3. CT scan with bone reconstruction, in which the arrow shows dehiscence of the anterior ethmoid artery, with a defect in the bone canal causing spontaneous rhinoliquorrhea.

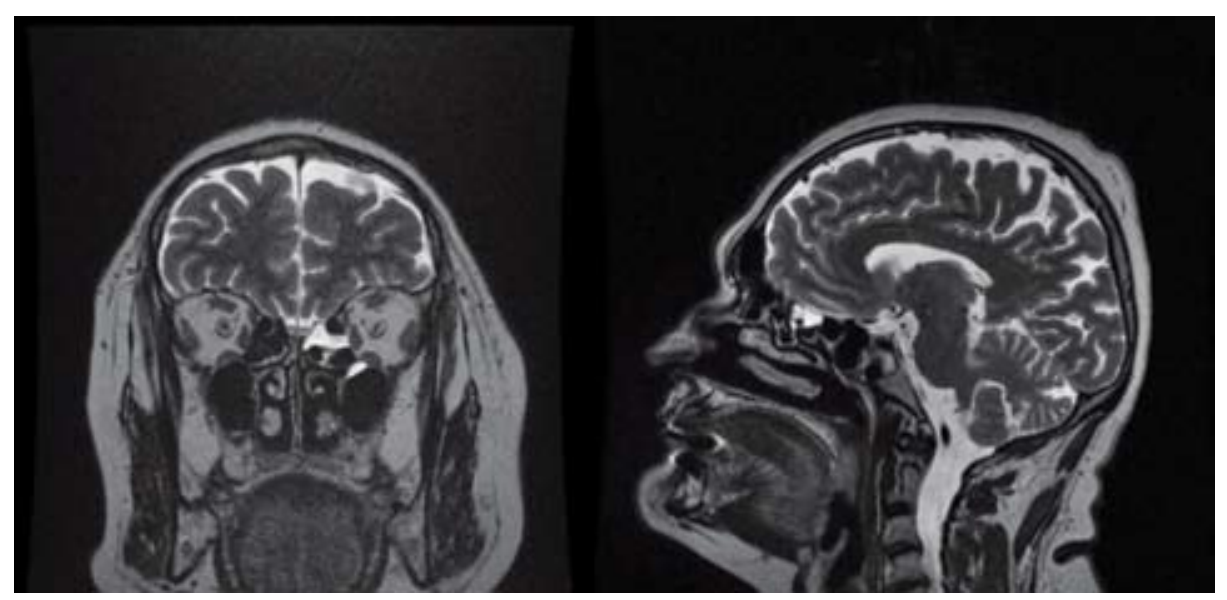

Figure 4. Coronal and sagittal view of the magnetic resonance imaging in the T2 sequence of the patient from Figure 3 shows a hyperintense signal in the area of the ethmoid roof. Intraoperatively confirmed localization. 


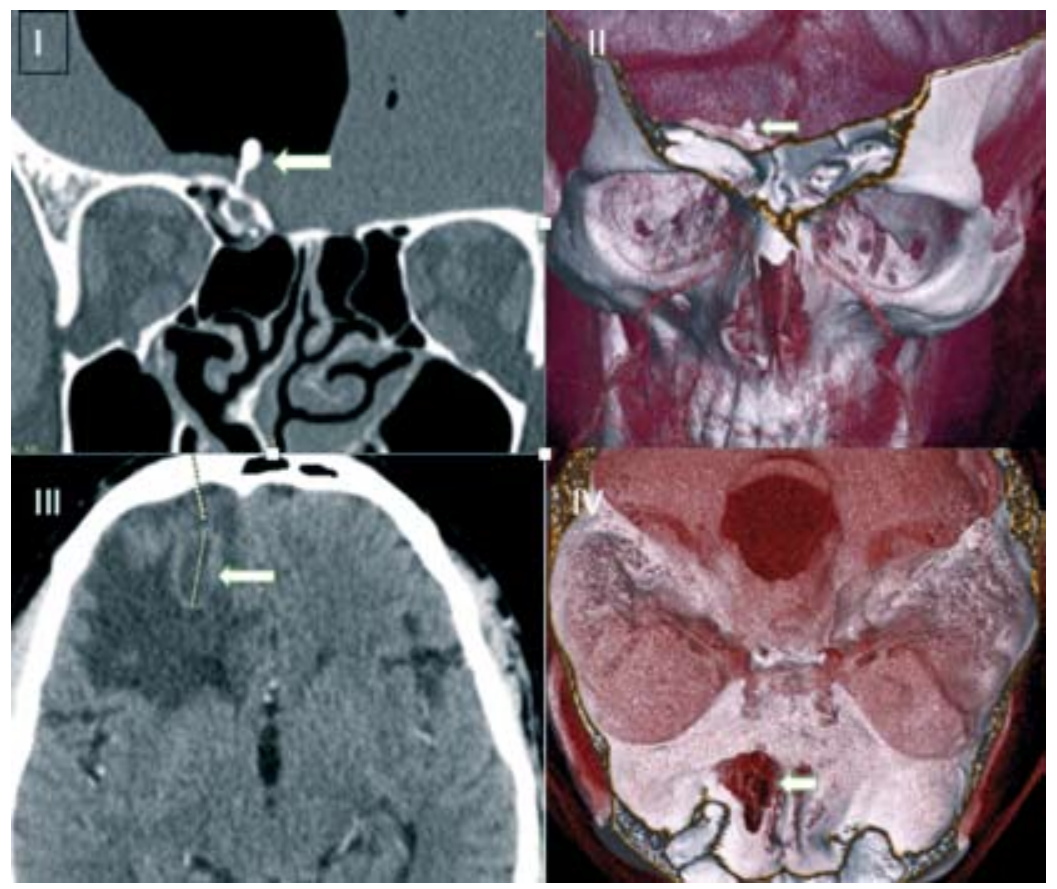

Figure 5. A case of an osteoma with spontaneous rhinoliquorrhea. A 28-year-old male with a debut of the disease - headache and feeling of heaviness in the left side of the face. Several months ago, he noticed a colorless secretion that is still leaking "in drops" from his nose. I - Coronal plane of HRCT with visualization of pneumocephalia and an osteoma in the area of the ethmoidal sinus. II - VR reconstruction with visualization of the bone spike of the osteoma. III - one month after surgery, HRCT axial plane with visualization of postoperative brain abscess frontobasally to the right. IV - VR reconstruction with a presentation of the postoperative defect in the area of the upper anterior base.

In some patients, when we have a clear defect proven with a CT scan, the application of fluorescein intrathecally is unjustified, with no indication for its use. In 14 of the cases, we used a combined approach in visualizing the localization of the defects in the rhinobase with the additional use of magnetic resonance imaging. The use of fluorescein, on the other hand, is part of the strategy to verify the successful repair of defects after duraplasty.

\section{Discussion}

The cerebrospinal fluid leak through the nasal cavity is due to a defect in the skull base area, in the bone or dural matter. The manifestation of rhinoliquorrhea may also be associated with defects in the posterior skull base. The ascendant pathway from the non-sterile bacterial sinonasal cavity to the sterile subarachnoid space leads to infections with severe intracranial complications.

Based on the causes for rhinoliquorrhea, it may be post-traumatic - due to skull-based surgery, endoscopic endonasal surgery, trauma of non-iatrogenic origin, or spontaneous - due to bone remodeling, inherent defects, dehiscence, bone canals, menin- goencephalocele in the skull base area. Post-radiation osteitis and osteomyelitis are also a prerequisite for spontaneous rhinoliquorrhea due to bone changes. The most common locations are lamina cribrosa, the sphenoid sinus, the area of the anterior ethmoidal artery, the frontal sinus and clivus.

Identifying the rhinoliquorrhea - having and choosing the right diagnostic imaging method is extremely difficult and raises many questions. There are false positives as well as false-negative results in the confirmatory tests with glucose test alone, as well as negative results from the computed tomography images that do not confirm the presence of bone defects or detect false localizations. The clinical judgment of the physician must be supported by proper biochemical tests (Beta-2 transferrin, Beta-trace), and the physician should be able to familiarize the radiologist with the definitive data on the presence of cerebrospinal fluid in the nasal cavity.

Anamnestically, the information we receive as a trauma in the past, recent surgical interventions in the nasal septum and paranasal cavities, pituitary surgery, road accidents, collision injuries, and 
weight gain in a short period, etc. can direct us that the one-sided leak of a clear secretion in "drops" cannot be related to allergic or vasomotor rhinitis. Any suspicion of rhinoliquorrhea should be verified with a highly specific biochemical test - Beta2 transferrin and/or Beta-trace protein, which is contained in the cerebrospinal fluid.

The choice of an imaging diagnostic method plays a particularly important role in the preoperative preparation and the planning of surgical behavior. Imaging studies include, in the first place, the highresolution computed tomography and magnetic resonance imaging/cisternography. In fact, each method carries insufficient defect information on its own because it is specific to a particular type of tissue density. The anatomical peculiarities of the rhinobase, the cases of multiple surgical interventions in the area of the base, conditions after neurosurgical interventions, numerous small defects, and meningocele all create considerable difficulties in the topical diagnosis. However, the combination of CT and MRI methods can jointly determine smallsize defects associated with meningocele or meningoencephalocele, tumors - osteoma, meningioma, "empty sella", as well as indicate the presence of intracranial hypertension.

In the absence of a detectable defect in imaging results, a positive biochemical test leads to the choice of using fluorescein intrathecally. The decision to use this type of dye is made based on ethically and legally supported informed consent presented along with all information on possible complications that may arise during the intervention. The method is highly specific, strictly indicated and requires excellent preparation by the anesthesiology team, technical equipment (blue filter during endoscopy), and well-trained medical specialists in postoperative care.

\section{Conclusion}

Rhinoliquorrhea is a socially significant condition, which requires timely recognition in clinical practice. Choosing a diagnostic method includes both one that determines the presence of a cerebrospinal fluid leak and one that defines the defect in the skull base. The diagnostic value of only one imaging method is debatable, due to the high specificity, small defects, dehiscence (meningocele, meningoencephalocele, dermoid cysts, conditions after transnasal pituitary surgery), persistent bone canals and fissures in the skull base area. The multi-diagnostic approach is of great clinical importance for the successful surgical treatment of rhinoliquorrhea.

\section{Bibliograhy:}

1. Schlosser RJ, Bolger WE. Management of multiple spontaneous nasal meningoencephaloceles. Laryngoscope. 2002 Jun;112(6): $980-985$.

2. Woodworth BA, Prince A, Chiu AG, et al. Spontaneous CSF leaks: a paradigm for definitive repair and management of intracranial hypertension. Otolaryngol Head Neck Surg. 2008 Jun; 138(6): 715-720. doi: 10.1016/j.otohns.2008.02.010.

3. Kaufman B, Nulsen FE, Weiss MH, Brodkey JS, White RJ, Sykora GF. Acquired spontaneous, nontraumatic normal-pressure cerebrospinal fluid fistulas originating from the middle fossa. Radiology. $1977 \mathrm{Feb}$; 122(2): 379-387

4. Lee TJ, Huang CC, Chuang CC, Huang SF. Transnasal endoscopic repair of cerebrospinal fluid rhinorrhea and skull base defect: ten-year experience. Laryngoscope. 2004 Aug; 114(8): 1475-1481.

5. Dzhambazov K., Markov S, Topalova A. CSF Fluid rhinorrhea-diagnostic methods review and clinical cases demonstration. International bulletin of Oto Rhino Laryngology 2015.

6. Wise SK, Schlosser RJ. Evaluation of spontaneous nasal cerebrospinal fluid leaks. Curr Opin Otolaryngol Head Neck Surg. 2007 Feb; 15(1): $28-34$.

7. Psaltis AJ, Schlosser RJ, Banks CA, Yawn J, Soler ZM. Asystematic review of the endoscopic repair of cerebrospinalfluid leaks. Otolaryngol Head Neck Surg. 2012 Aug; 147(2): 196-203. doi: 10.1177/0194599812451090.

8. McMains KC, Gross CW, Kountakis SE. Endoscopic management of cerebrospinal fluid rhinorrhea. Laryngoscope. 2004 Oct; 114(10): 1833-1837.

9. Purkey MT, Woodworth BA, Hahn S, Palmer JN, Chiu AG. Endoscopic repair of supraorbital ethmoid cerebrospinal fluid leaks. ORL J Otorhinolaryngol Relat Spec. 2009; 71(2): 93 98. doi:10.1159/000193219.

10. Meco C, Oberascher G, Arrer E, et al. Beta-trace protein test: new guidelines for the reliable diagnosis of cerebrospinal fluid fistula. Otolaryngol Head Neck Surg. 2003; 129(5): 508-517

11. Shetty PG, Shroff MM, Fatterpekar GM, Sahani DV, Kirtane MV. A retrospective analysis of spontaneous sphenoid sinus fistula: MR and CT findings. AJNR Am J Neuroradiol. 2000; 21: 337-342. 